\title{
Analysis of Edge Waves due to a Point Source in the Presence of a PEC Wedge
}

\author{
Behnam Ghassemiparvin * ${ }^{1}$, Ayhan Altintas ${ }^{1}$
}

Bilkent University, Ankara, Turkey. e-mail: behnam@ee.bilkent.edu.tr

\begin{abstract}
In this paper, we investigate the behavior of the field in the paraxial region of a perfectly conducting wedge and analyze the guiding effect of the wedge. It is observed that as the wedge angle increases, the guidance effect of the wedge decreases and the scattered field in the paraxial region is maximum for the half plane. In addition, interaction of the edge waves with a spherical impedance scatterer is investigated. It is found that for the backscattering case, as the impedance mismatch between the spherical boss and the free-space increases the scattered field due to the boss increases. However for the forward scattering case, impedance of the boss does not affect the scattered field significantly.
\end{abstract}

\section{Introduction}

The canonical problem of scattering from impedance objects at the edge of perfectly conducting wedge is of interest in developing propagation models, radar cross section (RCS) calculations. In [1]we have presented a dyadic Green's function (DGF) solution for the spherical impedance scatterer at the edge is presented. In this work we investigate the dyadic Green's function in the paraxial region in order to determine the edge guided waves and their interaction with the scatterer on the edge. This analysis can be considered as a basis to extent the scope of current high frequency techniques.

\section{Paraxial Region}

Geometry of the problem is shown in Figure 1. A PEC wedge with exterior angle $\gamma$ is considered which extends infinitely in the $z$ direction. One side of the wedge lies on the $x z$ plane. A spherical impedance scatterer with impedance of $\eta \quad$ is positioned at the edge of the PEC wedge. Position vectors, $\bar{R}=r \hat{r}$ and $\bar{R}^{\prime}=r^{\prime} \hat{r}^{\prime}$ denote the observation and the source locations, respectively.

The dyadic Green's function of the wedge and a spherical boss, $\overline{\bar{\Gamma}}_{W B}$, is derived in [1] where the DGF is expanded in terms of spherical vector wave functions. This solution is valid almost everywhere and gives accurate results in the paraxial region.

$$
\begin{gathered}
\overline{\bar{\Gamma}}_{W B}\left(\bar{R}, \bar{R}^{\prime}\right)=\overline{\bar{\Gamma}}_{W}\left(\bar{R}, \bar{R}^{\prime}\right)+\overline{\bar{\Gamma}}_{B}\left(\bar{R}, \bar{R}^{\prime}\right) \\
\overline{\bar{\Gamma}}_{W}\left(\bar{R}, \bar{R}^{\prime}\right)=\frac{\hat{r} \hat{r}}{k_{0}^{2}} \delta\left(\bar{R}-\bar{R}^{\prime}\right)+\frac{j \pi}{2 k_{0}}\left\{\begin{array}{l}
\sum_{m=0}^{\infty} \sum_{n=0}^{\infty} \frac{\bar{M}_{e \mu n}^{(4)}\left(k_{0} \bar{R}\right) \bar{M}_{e \mu n}^{(1)}\left(k_{0} \bar{R}^{\prime}\right)+\bar{N}_{o \mu n}^{(4)}\left(k_{0} \bar{R}\right) \bar{N}_{o \mu n}^{(1)}\left(k_{0} \bar{R}^{\prime}\right)}{Q_{\mu n}(\mu+n)(\mu+n+1)} \quad r \geq r^{\prime} \\
\sum_{m=0}^{\infty} \sum_{n=0}^{\infty} \frac{\bar{M}_{e \mu n}^{(1)}\left(k_{0} \bar{R}\right) \bar{M}_{e \mu n}^{(4)}\left(k_{0} \bar{R}^{\prime}\right)+\bar{N}_{o \mu n}^{(1)}\left(k_{0} \bar{R}\right) \bar{N}_{o \mu n}^{(4)}\left(k_{0} \bar{R}^{\prime}\right)}{Q_{\mu n}(\mu+n)(\mu+n+1)} \quad r \leq r^{\prime} . \\
\overline{\bar{\Gamma}}_{B}\left(\bar{R}, \bar{R}^{\prime}\right)=\sum_{m=0}^{\infty} \sum_{n=0}^{\infty}\left\{\frac{\alpha_{\mu n}\left(k_{0} a\right) \bar{M}_{e \mu n}^{(4)}\left(k_{0} \bar{R}^{\prime}\right) \bar{M}_{e \mu n}^{(4)}\left(k_{0} \bar{R}\right)+\beta_{\mu n}\left(k_{0} a\right) \bar{N}_{o \mu n}^{(4)}\left(k_{0} \bar{R}^{\prime}\right) \bar{N}_{o \mu n}^{(4)}\left(k_{0} \bar{R}\right)}{Q_{\mu n}(\mu+n)(\mu+n+1)}\right\}
\end{array}\right.
\end{gathered}
$$

The dyadic Green's function of the wedge and boss consists of two parts; $\overline{\bar{\Gamma}}_{W}$, given in (2), is the DGF of the PEC wedge which is identical to the expression given in [2]. $\overline{\bar{\Gamma}}_{B}$, given in (3), is defined as the DGF of the boss which includes the added terms due to the presence of the spherical boss. $\bar{M}$ and $\bar{N}$ are spherical vector wave functions defined in [8]. Since the field should be regular at the origin, spherical Bessel functions denoted by superscript (1) are 


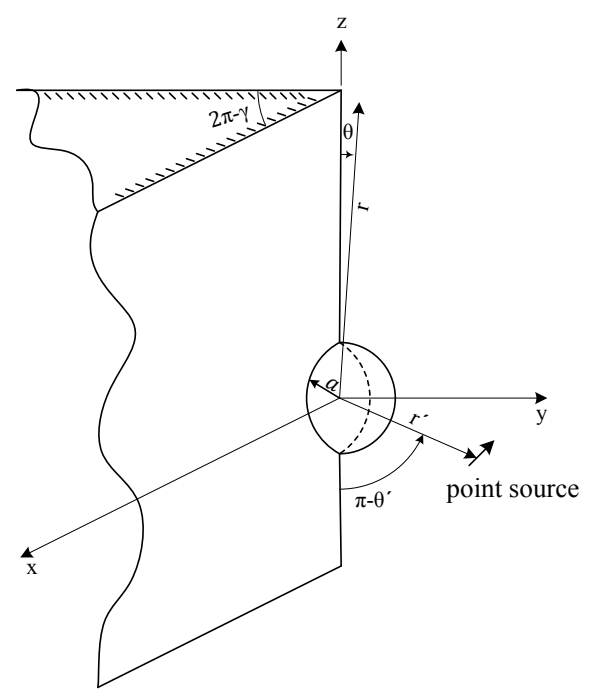

Figure 1 Geometry of the problem.

used. Hankel functions, denoted by superscript(4), are chosen to satisfy the radiation condition for the scattered field. $\mu=\frac{m \pi}{\gamma}$ are the eigenvlaues chosen to satisfy the PEC boundary condition on the surface of the wedge. The coefficients $\alpha_{\mu n}\left(k_{0} a\right)$ and $\beta_{\mu n}\left(k_{0} a\right)$ are defined in [1] which are related to the impedance $\kappa=\frac{\eta}{j k_{0} Z_{0}}$ and the radius of the impedance boss, $a$.

As mentioned in [1-2] in the paraxial region scattered field is highly enhanced due to the edge guided waves and the dominant mode is $m=1, n=0$. We will use only this term for approximating the fields in the paraxial region.

To evaluate the field in the paraxial region we assume that $\theta \rightarrow 0$ and $k_{0} r \rightarrow \infty$ therefore we use the far-field approximation for the observation point. Considering only the $m=1, n=0$ term we can write the dyadic Green's function for the spherical boss as,

$$
\begin{gathered}
\overline{\bar{\Gamma}}_{B}\left(\bar{R}, \bar{R}^{\prime}\right)=C(\gamma) G_{F}(\bar{R}) G_{s}^{B}\left(\bar{R}^{\prime}\right) \\
C(\gamma)=\frac{\Gamma\left(\frac{2 \pi}{\gamma}+2\right) e^{j \frac{\pi}{2}(\pi / \gamma+1)}}{2^{2 \pi / \gamma} \gamma \Gamma\left(\frac{\pi}{\gamma}\right) \Gamma\left(\frac{\pi}{\gamma}+1\right)} \\
\bar{G}_{F}(\bar{R})=\frac{e^{-j k_{0} z}}{z^{\pi / \gamma} \rho^{1-\pi / \gamma}}\left[\hat{a}_{\rho} \sin \left(\frac{\pi}{\gamma} \phi\right)+\hat{a}_{\phi} \cos \left(\frac{\pi}{\gamma} \phi\right)\right] \\
G_{s}^{B}\left(\bar{R}^{\prime}\right)=\frac{1}{\sin ^{1-\pi / \gamma}\left(\theta^{\prime}\right)}\left\{\hat{a}_{r^{\prime}} \beta_{\pi / \gamma, 0}\left(k_{0} a\right) \frac{\mathrm{h}_{\pi / \gamma}^{(2)}\left(k_{0} r^{\prime}\right)}{k_{0} r^{\prime}} \sin \left(\theta^{\prime}\right) \sin \left(\frac{\pi}{\gamma} \phi^{\prime}\right)\right. \\
+\frac{\hat{a}_{\theta^{\prime}}}{(\pi / \gamma+1)}\left[\alpha_{\pi / \gamma, 0}\left(k_{0} a\right) j \mathrm{~h}_{\pi / \gamma}^{(2)}\left(k_{0} r^{\prime}\right)+\beta_{\pi / \gamma, 0}\left(k_{0} a\right) \frac{1}{k_{0} r^{\prime}} \frac{d}{d r^{\prime}}\left[r^{\prime} \mathrm{h}_{\pi / \gamma}^{(2)}\left(k_{0} r^{\prime}\right)\right] \cos \left(\theta^{\prime}\right)\right] \sin \left(\frac{\pi}{\gamma} \phi^{\prime}\right) \\
+\frac{\hat{a}_{\phi^{\prime}}}{(\pi / \gamma+1)}\left[\alpha_{\pi / \gamma, 0}\left(k_{0} a\right) j \mathrm{~h}_{\pi / \gamma}^{(2)}\left(k_{0} r^{\prime}\right) \cos \left(\theta^{\prime}\right)+\beta_{\pi / \gamma, 0}\left(k_{0} a\right) \frac{1}{k_{0} r^{\prime}} \frac{d}{d r^{\prime}}\left[r^{\prime} \mathrm{h}_{\pi / \gamma}^{(2)}\left(k_{0} r^{\prime}\right)\right] \cos \left(\frac{\pi}{\gamma} \phi^{\prime}\right)\right\}
\end{gathered}
$$

Dyadic Green's function of the PEC wedge can also be written as,

$$
\overline{\bar{\Gamma}}_{W}\left(\bar{R}, \bar{R}^{\prime}\right)=C(\gamma) G_{F}(\bar{R}) G_{s}^{W}\left(\bar{R}^{\prime}\right)
$$

where 


$$
\begin{aligned}
G_{s}^{W}(\bar{R}) & =\frac{1}{\sin ^{1-\pi / \gamma}\left(\theta^{\prime}\right)}\left\{\hat{a}_{r^{\prime}} \frac{\mathrm{j}_{\pi / \gamma}\left(k_{0} r^{\prime}\right)}{k_{0} r^{\prime}} \sin \left(\theta^{\prime}\right) \sin \left(\frac{\pi}{\gamma} \phi^{\prime}\right)\right. \\
& +\frac{\hat{a}_{\theta^{\prime}}}{(\pi / \gamma+1)}\left[j \mathrm{j}_{\pi / \gamma}\left(k_{0} r^{\prime}\right)+\frac{1}{k_{0} r^{\prime}} \frac{d}{d r^{\prime}}\left[r^{\prime} \mathrm{j}_{\pi / \gamma}\left(k_{0} r^{\prime}\right)\right] \cos \left(\theta^{\prime}\right)\right] \sin \left(\frac{\pi}{\gamma} \phi^{\prime}\right) \\
& +\frac{\hat{a}_{\phi^{\prime}}}{(\pi / \gamma+1)}\left[j \mathrm{j}_{\pi / \gamma}\left(k_{0} r^{\prime}\right) \cos \left(\theta^{\prime}\right)+\frac{1}{k_{0} r^{\prime}} \frac{d}{d r^{\prime}}\left[r^{\prime} \mathrm{j}_{\pi / \gamma}\left(k_{0} r^{\prime}\right)\right] \cos \left(\frac{\pi}{\gamma} \phi^{\prime}\right)\right\}
\end{aligned}
$$

$C(\gamma)$ only depends on the wedge angle. According to (6), field varies as $\cos \left(\frac{\pi}{\gamma} \phi\right)$ and $\sin \left(\frac{\pi}{\gamma} \phi\right)$ for $\hat{a}_{\phi}$ and $\hat{a}_{\rho}$ components, respectively. Therefore the scattering pattern in the paraxial region is determined by the wedge angle. As the wedge angle increases ( $\gamma$ decreases), field attenuates with higher powers of the inverse of the distance $z$. $G_{s}^{W}$ depends only on the source position and $G_{s}^{B}$ is also related to the properties of the scatterer.

\section{Numerical Results}

To investigate the effects of the wedge angle on the electric field in the paraxial region, scattered field of a PEC wedge is plotted for different wedge angle. A point source positioned near the PEC wedge (without a boss) and the observation point is in the paraxial region. Source and observation points are both close to the wedge but they are separated by a large distance. Source is a $\hat{a}_{z}$ directed infinitesimal dipole positioned at $\theta^{\prime}=\pi / 2$ and $r^{\prime}=0.1 \lambda$ and $\phi^{\prime}=\gamma / 2$. Observation point is at $z=10 \lambda, \rho=0.1 \lambda$.

Figure 2 shows the scattered field components for this case. As expected the scattered field intensity decreases as wedge angle increases and the guiding effect of the wedge is maximum for a half plane $(\mathrm{WA}=0)$.

In addition dyadic Greens function of the boss, $\overline{\bar{\Gamma}}_{B}$, is evaluated for a $\hat{a}_{\theta}$ directed point source positioned at $\theta^{\prime}=1^{\circ} \quad r^{\prime}=1 \lambda$ with a spherical scatterer of radius $a=\lambda / 4$ is considered on the edge. Observation point is at $z=10 \lambda, \rho=0.1 \lambda$. For this configuration, scattered field due to the spherical boss, $E_{B}$, and the total field $E=E_{B}+E_{W}$ is plotted in Figure 3.
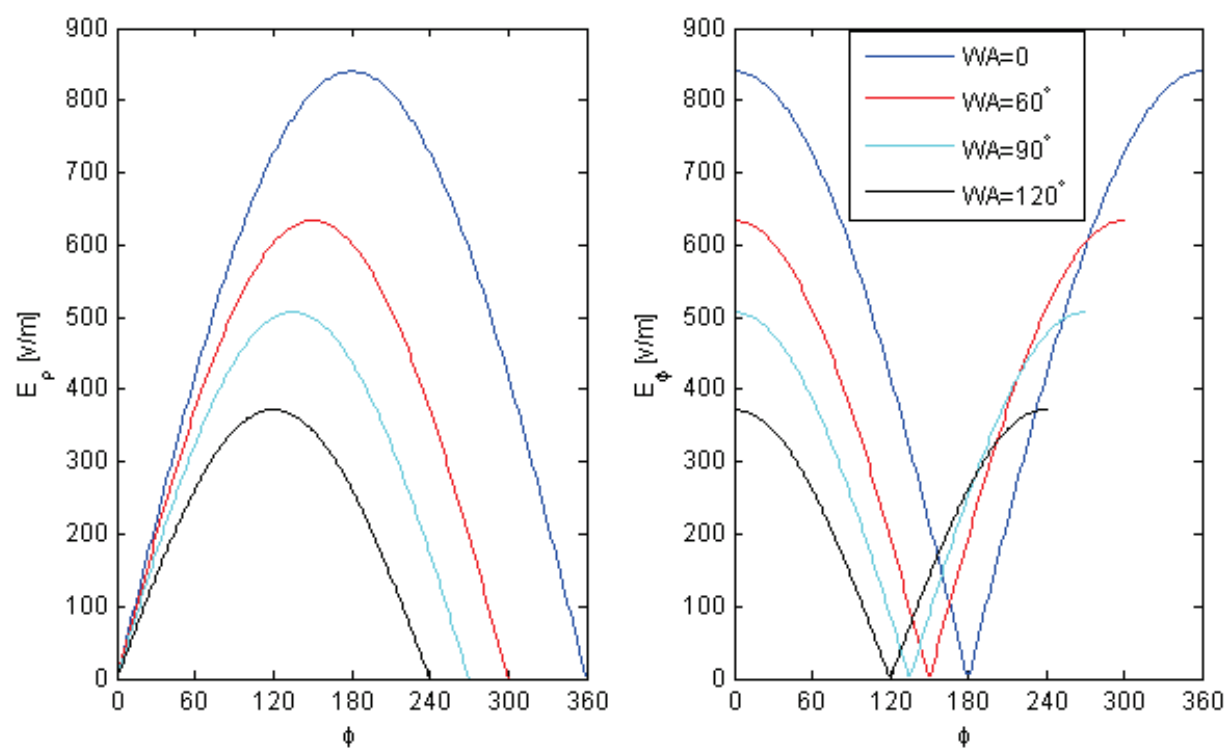

Figure 2 Scattered field due to a $\hat{a}_{z}$ directed point source at $\theta^{\prime}=\pi / 2$ and $r^{\prime}=0.1 \lambda$ and $\phi^{\prime}=\gamma / 2$. Observation point is at $z=10 \lambda, \rho=0.1 \lambda$ 

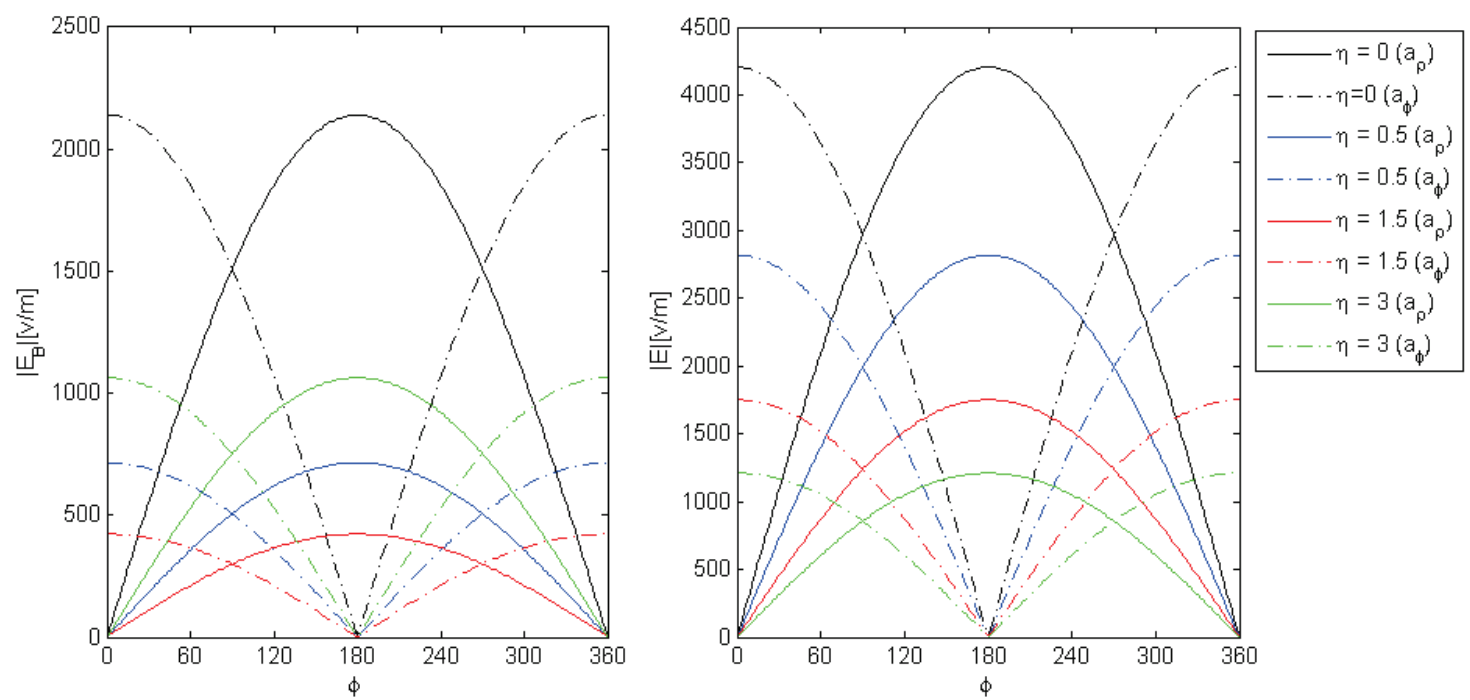

Figure 3 Scattered field due to a $\hat{a}_{z}$ directed point source at $\theta^{\prime}=1^{\circ}$ and $r^{\prime}=1 \lambda$ and $\phi^{\prime}=\gamma / 2$. Observation point is at $z=10 \lambda, \rho=0.1 \lambda$ (a) Scattered field due to the boss (b) Total field

Figure 3 shows that as the impedance mismatch between the spherical scatterer and free-space increases, scattered field due to the scatterer increases. Based on these results, it is seen that for $\eta / Z_{0}<1$ edge waves, $E_{W}$ interfere destructively with the $E_{B}$ and the total field increases. But for $\eta / Z_{0}>1$ it is observed that the fields interfere destructively so the total field decreases for larger $\eta$ values.

It can be shown for the $\theta^{\prime} \rightarrow \pi$, the scattered field amplitude does not vary significantly with respect to the impedance.

\section{Conclusion}

A simple dyadic Green's function expression is derived for the canonical problem of a spherical impedance boss at the edge which is valid only in the paraxial region. Effect of the wedge angle on the guiding effect of the wedge is investigated and it is observed that the scattered field is maximized for the half plane case. Interaction of the edge waves with a spherical impedance scatterer is also considered. It is seen that the total scattered field due to the spherical boss increases as the impedance mismatch between the scatterer and free-space increases.

\section{References}

1. B. Ghassemiparvin, and A. Altintas. "Scattering from an Impedance Object at the Edge of a Perfectly Conducting Wedge.” IEEE Trans. Antennas Propag., vol. 62, no. 2, pp.852-861, 2014.

2. O. M. Buyukdura, S. D. Goad, and R. G. Kouoyoumjian, "A spherical wave representation of the dyadic Green's function for a wedge," IEEE Trans. Antennas Propag., vol. 44, no. 1, pp. 12-22, 1996. 